\title{
Phenotypic characterization of three clinical isolates of Burkholderia pseudomallei in Ceará, Brazil
}

\author{
CG Virginio/ ${ }^{+}$, MFS Teixeira*, CC Frota, VS Café, MFG Rocha, JJC Sidrim
}

Departamento de Patologia e Medicina Legal, Centro Especializado em Micologia Médica, Universidade Federal do Ceará, Rua Monsenhor Furtado s/nº, 60441-750 Fortaleza, CE, Brasil *Faculdade de Veterinária, Pós Graduação em Ciências Veterinárias, Universidade Estadual do Ceará, Fortaleza, CE, Brasil

Burkholderia pseudomallei, the causative agent of melioidosis was found in a small cluster of cases in Tejuçuoca, Ceará, Brazil. Tests were carried out to determine its phenotypic characteristics: colony morphology on Ashdown agar and MacConkey agar, biochemical profile in conventional biochemical tests and API 20NE, arabinose assimilation and susceptibility testing by disk diffusion, comparing with data in the literature. This study confirms the presence of B. pseudomallei in Brazil and describes its characteristics.

Key words: Burkholderia pseudomallei - melioidosis - Brazil

Melioidosis is a serious human disease caused by Burkholderia pseudomallei. Isolation of B. pseudomallei from human samples had not been reported in Brazil until 2003 (Miralles et al. 2004, Braga \& Almeida 2005). The purpose of this study was to compare the characteristics of these Brazilian isolates of the species with others reported in the literature, and to alert microbiologists in the region to these characteristics in order to enable them to recognize subsequent cases.

In this study three isolates of $B$. pseudomallei were analyzed. These were isolated from clinical samples from a small cluster of cases that occurred in Tejuçuoca, Ceará, Brazil, involving three patients from the same family, aged 10-15 years old. Two strains of $B$. pseudomalle $i$ were initially isolated on MacConkey agar from the spleen, liver, and lung and one strain from blood culture in BHI broth. All procedures involving viable cells were performed in a category 3 containment laboratory.

Strains were identified phenotipically according to the criteria described for gram-negative nonfermentative bacilli and by characteristics on Ashdown selective medium (Ashdown 1979, Gilligan \& Whittier 1999, Koneman et al. 2001). The identification of B. pseudomallei was confirmed by biochemical tests using the API 20NE system (bioMérieux) (Dance et al. 1989).

The bacteria were short, motile bipolar gram-negative bacilli. All three isolates showed characteristic bipolar staining, probably because of intracellular deposits of $\beta$ hydroxy butyric acid (Inglis et al. 2001, Sprague \& Neubauer 2004). A thick and dry pellicle with a matt surface was seen on BHI broth cultures. This pellicle forms at the broth-air interface, possibly because of positive

Financial support: Capes

+Corresponding author: camila.virginio@ @ecrel.com.br

Received 11 May 2005

Accepted 9 January 2006 aerotaxis of B. pseudomallei, and reflects a type of a multicellular organization that resembles a biofilm.

On MacConkey agar after $24 \mathrm{~h}$ of incubation the colonies were pink-transparent, small, smooth, with sheen and convex. After seven days of culture, isolates 1 and 3 showed larges, circulars, viscous, with sheen on surface and entire borders colonies. Isolate 2 also produced circulars, with surface sheen and pink colonies, however showed consistence dry and wrinkled.

Ashdown's selective agar is commonly used to culture the organism from a mixed flora. All the B. pseudomallei isolates had characteristic colonial morphology on Ashdown agar. The colonies were smooth, moist, purple in color, dome shaped, about 0.8 to $1 \mathrm{~mm}$ in diameter, on plates after $24 \mathrm{~h}$ of incubation at $37^{\circ} \mathrm{C}$. After seven days of incubation isolate 2 grew as purple colonies, wrinkled, 5 to $6 \mathrm{~mm}$ in diameter, which had centrally umbonated, with radiating ridges at the periphery and a characteristic earthy odor; this showed a typical colonial morphology (Ashdown 1979). Isolates 1 and 3 included both smooth and mucoid colonies. The isolation of mucoid variant strains from clinical specimens is relatively uncommon (Inglis et al. 2001). Howard and Inglis (2003) have suggested that this may reflect inhibitory effect of crystal violet in Ashdown's agar. However, this not was observed in isolates 1 and 3 .

B. pseudomallei and a closely related species Burkholderia thailandensis, can be distinguished by their ability to assimilate the aldopentose L-arabinose. The Larabinose non assimilators, $B$. pseudomallei, are highly virulent and can be isolated from both clinical specimens and the environment. The L-arabinose assimilators, now known as $B$. thailandensis, however, are generally avirulent and found predominantly in the environment (Brett et al. 1998). B. pseudomallei isolates were tested for arabinose assimilation by growth on minimal salts agar containing 0.2\% L-arabinose (Wuthiekanun et al. 1996). None of the clinical isolates of $B$. pseudomallei could assimilate arabinose, which was demonstrated by absence of growth on minimal salts medium in $48 \mathrm{~h}$. Although Ara $+B$. pseudomallei has been reported to cause infection 
(Lertpatanasuwan et al. 1999), all isolates causing melioidosis in our study were Ara-. Our findings support previous observations described by Vuddhakul et al. (1999) in Thailand and by Miralles et al. (2004) in Brazil. In this study, the results of the biochemical profile in the OF medium tests with lactose, maltose, and mannitol for isolates 1 and 3 differed from those Gilligan and Whittier (1999), but this may reflect differences in methodology utilized for assimilation tests, rather than genuine differences in Brazilian isolates.

All the B. pseudomallei isolates were identified correctly by API $20 \mathrm{NE}$ in $48 \mathrm{~h}$. The profile obtained for isolates 1, 2, and 3 were $\mathbf{1 5 5 6 5 7 7}$ (99.1\% correct identification), $\mathbf{1 1 5 6 5 7 7}$ and $\mathbf{1 1 5 6 5 7 7}$ (99.9\% correct identification) respectively. In API 20NE strips, differences in esculin hydrolysis were observed (isolate 1 was positive, isolates 2 and 3 were negative), although this did not affect the identification profile, as esculin hydrolysis by $B$. pseudomallei is quite variable (Gilligan \& Whittier 1999, Koneman et al. 2001).

Susceptibility tests were undertaken with the following antibiotics: piperacilin-tazobactam, ticarcilinclavulanate, ceftazidime, ceftriaxone, cephalotin, imipenem, gentamicin, tetracycline, ciprofloxacin, chloramphenicol, and sulfamethoxazole-trimethoprim (Cecon $\left.{ }^{\circledR}\right)$. All antibiotics were tested by disk diffusion using standard methods (NCCLS 2003). The results of susceptibility tests are shown in Table and were typical of B. pseudomallei. In general, the Brazilian isolates exhibited similar profile to those other parts of the world. Our report confirms previous studies as realized by Gales et al. (2005) for in vitro susceptibility Burkholderia species. However, in this study, our isolates were resistant to sulfamethoxazoletrimethoprim, although this may have occurred by differences in methodology utilized. B. pseudomalle $i$ is known to produce a number of beta-lactamases (Niumsup \& Wuthiekanun 2002). These results mean that the current recommendations for the treatment of melioidosis can also be applied to case from Brazil (CDC 2004).

TABLE

Antibiotic susceptibility patterns of three isolates of Burkholderia pseudomallei determined by disk diffusion

\begin{tabular}{lcccc}
\hline & & \multicolumn{3}{c}{ Isolates } \\
\cline { 3 - 5 } Antimicrobial agent & Disk $(\mu \mathrm{g})$ & 1 & 2 & 3 \\
\hline Piperacilin-tazobactam & $100 / 10$ & $\mathrm{~S}$ & $\mathrm{~S}$ & $\mathrm{~S}$ \\
Ticarcilin-clavulanate & $75 / 10$ & $\mathrm{~S}$ & $\mathrm{~S}$ & $\mathrm{~S}$ \\
Ceftazidime & 30 & $\mathrm{~S}$ & $\mathrm{~S}$ & $\mathrm{~S}$ \\
Ceftriaxone & 30 & $\mathrm{I}$ & $\mathrm{I}$ & $\mathrm{S}$ \\
Cefalotin & 30 & $\mathrm{R}$ & $\mathrm{R}$ & $\mathrm{R}$ \\
Imipenem & 10 & $\mathrm{~S}$ & $\mathrm{~S}$ & $\mathrm{~S}$ \\
Gentamicin & 10 & $\mathrm{R}$ & $\mathrm{R}$ & $\mathrm{R}$ \\
Tetracycline & 30 & $\mathrm{~S}$ & $\mathrm{~S}$ & $\mathrm{~S}$ \\
Ciprofloxacin & 5 & $\mathrm{I}$ & $\mathrm{R}$ & $\mathrm{R}$ \\
Chloramphenicol & 30 & $\mathrm{~S}$ & $\mathrm{~S}$ & $\mathrm{~S}$ \\
Sulfa-trimethoprim & 25 & $\mathrm{R}$ & $\mathrm{R}$ & $\mathrm{R}$ \\
\hline
\end{tabular}

R: resistant; I: intermediate; S: susceptible

\section{ACKNOWLEDGEMENTS}

To Universidade Federal do Ceará, Department of Microbiology, Faculty of Medicine, for technical support.

\section{REFERENCES}

Ashdown LR 1979. An improved screening for isolation of Pseudomonas pseudomallei from clinical specimens. $\mathrm{Pa}$ thology 11: 293-297.

Braga MD, Almeida PRC 2005. Primeira descrição de um caso autopsiado de melioidose no Estado do Ceará. Rev Soc Bras Med Trop 38: 58-60.

Brett PJ, Deshazer D, Woods DE 1998. Burkholderia thailandensis sp. nov., a Burkholderia pseudomallei-like species. Int J Syst Bacteriol 48: 317-320.

CDC-Centers for Disease Control and Prevention 2004. Laboratory exposure to Burkholderia pseudomallei-Los Angeles, California, 2003. MMWR 53: 988-990.

Dance DAB, Wuthiekanun V, Naigowit P, White NJ 1989. Identification of Pseudomonas pesudomallei in clinical practice: use of simple screening tests and API 20NE. J Clin Pathol 42: 645-648.

Gales AC, Jones RN, Andrade SS, Sader HS 2005. Antimicrobial susceptibility patterns of unusual nonfermentative gramnegative bacili isolated from Latin America: report from the SENTRY Antimicrobial Surveillance Program (1997-2002). Mem Inst Oswaldo Cruz 100: 571-577.

Gilligan PH, Whittier S 1999. Miscellaneous Gram-negative bacteria. In PR Murray, EJ Baron, MA Pfaller, FC Tenover, RH Yolken (eds), Manual of Clinical Microbiology, 7th ed., American Society for Microbiology, US, p. 526-538.

Howard K, Inglis TJJ 2003. Novel selective medium for isolation of Burkholderia pseudomallei. J Clin Microbiol 41: 3312-3316.

Inglis TJJ, Mee BJ, Chang BJ 2001. The environmental microbiology of melioidosis. Rev Med Microbiol 12: 13-20.

Koneman EW, Allen MD, Janda WM, Schreckenberger PC 2001. Diagnóstico Microbiológico, $5^{a}$ ed., Medsi, Rio de Janeiro, $1465 \mathrm{pp}$.

Lertpatanasuwan N, Sermsri K, Petkaseam A, Trakulsolboon S, Thamlikitkul V, Suputtamongkol Y 1999. Arabinose-positive Burkholderia pseudomallei infection in humans: case report. Clin Infect Dis 28: 927-928.

Miralles IS, Maciel MCA, Angelo MRF, Gondini MM, Frota LHF, Reis CMF, Hofer E 2004. Burkholderia pseudomallei: a case report of a human infection in Ceará, Brazil. Rev Inst Med Trop São Paulo 46: 51-54.

NCCLS-National Committee for Clinical Laboratory Standards 2003. Performance Standards for Antimicrobial Disk Susceptibility Test (approved standard M2-A8), 8th ed., Wayne.

Niumsup P, Wuthiekanun V 2002. Cloning of class D betalactamase gene from Burkholderia pseudomallei and studies on its expression in ceftazidime-susceptible and-resistant strains. J Antimicrob Chemother 50: 445-455.

Sprague LD, Neubauer H 2004. Melioidosis in animals: a rewiew on epizootiology, diagnosis and clinical presentation. $J$ Vet Med 51: 305-320. 
Vuddhakul V, Tharavichitkul P, Na-Ngam N, Jitsurong S, Kunthawa B, Noimay P, Binla A, Thamlikitkul V 1999. Epidemiology of Burkholderia pseudomallei in Thailand. Am J Trop Med Hyg 60: 458-461.
Wuthiekanun V, Smith MD, Dance DAB, Walsh AL, Pitt TL, White NJ 1996. Biochemical characteristics of clinical and environmental isolates of Burkholderia pseudomallei.J Med Microbiol 45: 408-412. 